\title{
Research on the Deformation Behavior of Radial Rotary Forging with Cylindrical Spur Gear
}

\author{
Wen Yong $\mathrm{Shi}^{1,2,3}$, Yan $\mathrm{Ma}^{2}$ and $\mathrm{Zhe} \mathrm{Wu}^{2}$ \\ ${ }^{1}$ Division of Graduate, Harbin University of Science and Technology, Harbin \\ 150080, P R China \\ ${ }^{2}$ Forest Woodworking Machinery Research and Development Center, Northeast \\ Forestry University, Harbin 150040, P R China \\ ${ }^{3}$ Key Laboratory of Superlight Materials and Surface Technology, Harbin \\ Engineering University, Ministry of Education, Harbin 150001, P R China
}

\begin{abstract}
Reducing energy consumption and improving the quality performance of products are the important goals in the precise forming field of gear. Because of the requirement of precision, the traditional gear forging technology needs to be improved. Compared with traditional gear forging technology, the radial rotary forging technique can change the loading mode. Namely, the integral loading method replaces the local loading method. The forming load can be significantly reduced and the strength of the gear can be improved. During the deformation process, the flowing of material turns to be uniform. Therefore, the quality of gear produced by radial rotary forging is significantly improved. In a word, the radial rotary forging could provide a scientific basis for the research of high performance gear with a high efficiency and low consumption.
\end{abstract}

Key words: Cylindrical spur gear; Rotary forging; Loading mode

\section{Introduction}

Gear is one of the important parts in the automobile gearbox and all kinds of mechanical power transmission systems. The quality of gear has a decisive influence on the stability and reliability of the whole running system. Therefore, the research of gear machining method and the improvement of gear's comprehensive performance has always been focused in the industry [1-2]. Traditional gear manufacturing mainly relies on machining, but the bottleneck (low material utilization rate, low production efficiency and big energy consumption.) is difficult to meet the actual demand of the current production. Due to the high demand of product, the existing gear precision forging technique still needs to be improved [3]. The material has good formability, when the precision spur gears are forged. But large energy consumption, low material utilization ratio and the precision are difficult to be guaranteed [4]. Cold forging method can obtain higher precision and smaller energy consumption, but it has a large deformation resistance to form. A flow dividing method can reduce the forming load and improve the material filling ability, but the forming process is more complicated. The way to realize the high efficient and low consumption is still to be explore in the current gear machining and forming field [5-6]. Due to the above problems, this paper is to study the change of billet shape and the filling behavior during the gear rotary forging process. At the same time, the advantages and disadvantages of rotary forging forming technique and the precision forging technique are compared. 


\section{The Process Principle and Scheme}

\subsection{Manufacturability Analysis}

The principle of the gear rotary forging process is shown in Figure 1. First, the billet needs to be prefabricated into circular ring and put on the core shaft. The rotary forging die is driven by the transmission device to do clockwise or anticlockwise rotation, and the rotation angle is $1 /$ ( $2 n$ ) circle ( $n$ represents the number of teeth). Convex on the inside of the rotary forging die pushes the punch. The punch moves gradually along the radial direction to the center of the circle in the guide appliance. And the plastic deformation occurs on the surface of the ring billet. The billet between two adjacent punches produces convex tooth under compression deformation. Then it continues to $1 /(2 \mathrm{n})$ circle clockwise or anticlockwise. The punch gets back to the concave part of the inside of rotary forging die, and then the forming of gear parts can be taken out.

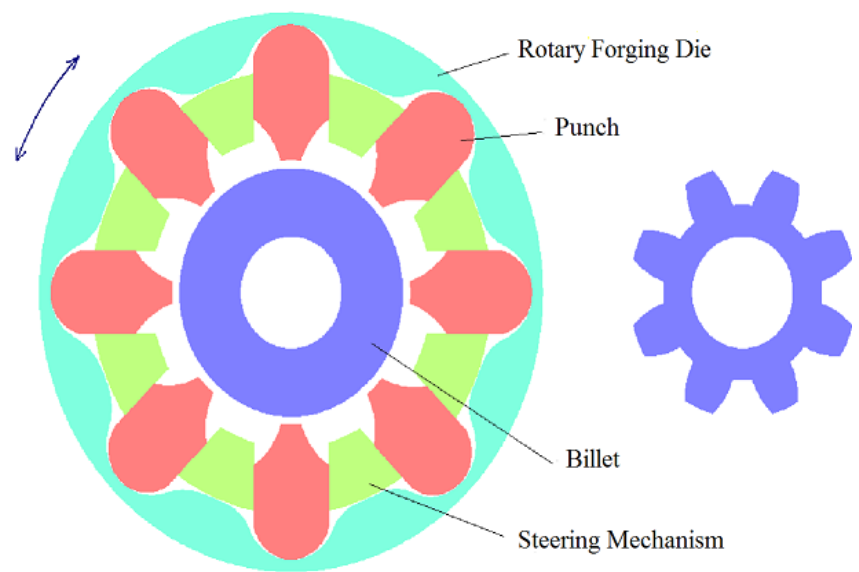

(a)Process principle schematic

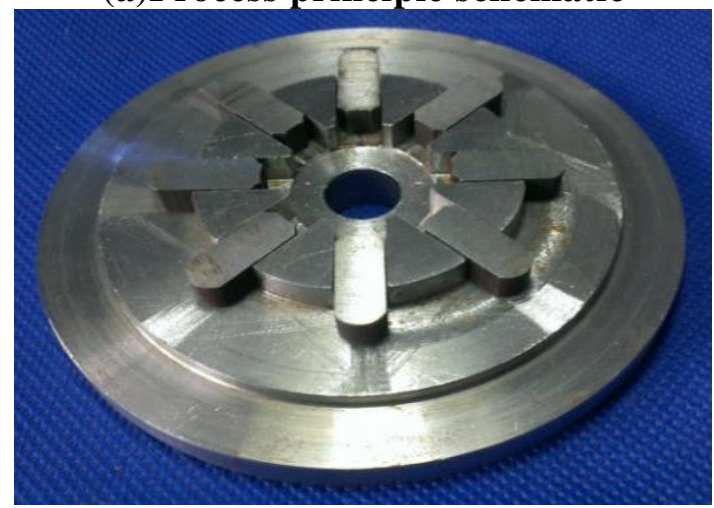

(b)Mould photos

\section{Figure 1. Schematic Diagram of the Rotary Forging Process}

As is shown in Figure 1, this method can solve the problem that the feet cavity is difficult to be filled fully due to the effect of friction on gear precision forging technology, because loading mode is changed and gear forming plastic deformation area is concentrated on the surrounding of the convex tooth. The required size can be met. In addition, it also can strengthen the intensity of gear. At the same time, according to the requirement of gear structure and size, we can respectively change the punch, steering mechanism and the structure of the rotary forging die which can achieve the goal of producing different module of gear process. In order to make the research convenient, we chosen $=8$. 


\subsection{Finite Element Model}

The research uses finite element simulation software DEFORM-3D to analyze the process of gear rotary forging. We choose Steel 20 as the research material. During the process of simulation, we ignore the elastic deformation and use rigid-plastic finite element model for analysis. Four nodes tetrahedron is oparametric element is chosen to discrete the billet. In order to reduce the computing workload, only $1 / 8$ of the billet is employed for simulation. The iteration algorithm is Newton-Rap son and the adaptive weight division is used in the meshing process. The finite element model is shown in Figure 2. The settings of meshing and symmetry plane are shown in Figure 2 (b).

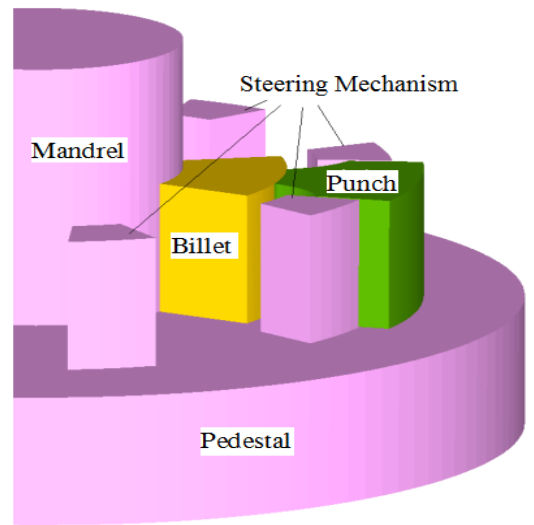

(a) Finite element model

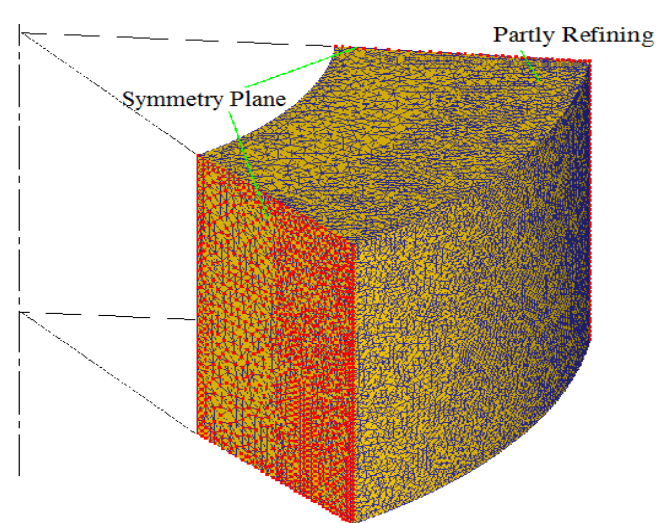

(b) Meshing

Figure 2. Finite Element Model

Forming process is conducted at room temperature. The Rotary forging angular velocity is $0.032 \mathrm{rad} / \mathrm{s}$. Friction type between billet and mold is defined as the shear friction model, and friction factor is $m=0.12$. The inner and outer diameter and the height of the initial ring billet are $6 \mathrm{~mm}, 10.25 \mathrm{~mm}$ and $6 \mathrm{~mm}$, respectively. The speed of the punch is $1 \mathrm{~mm} / \mathrm{s}$. Other conditions are the same as the rotary forging process.

\section{Discussion and Analysis}

\subsection{Mould Filling Process}

The billet deformation and filling behavior during the rotary forging forming is shown in Figure 3.

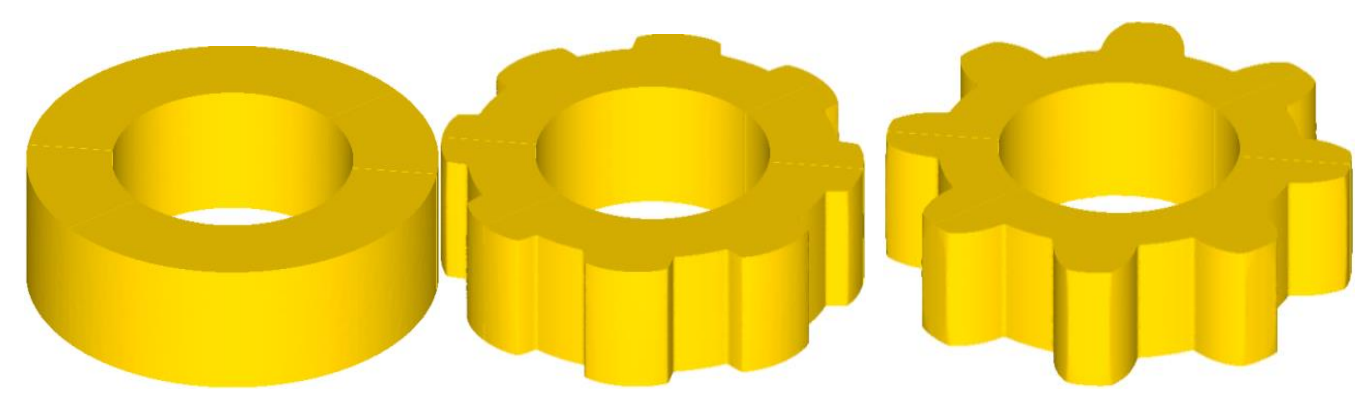

Figure 3. The Billet Deformation Characteristic under Rotary Forging Technological Condition

Figure 3 describes the billet deformation characteristics in the process of rotary forging. As we can see, billet along the height direction has no change in the whole loading 
process. Eight punches are driven by the rotary forging die. At this point, the cavity can be filled simultaneously. At the early stage of precision forging, the ring billet under the compression deformation moves toward outside. With the movement of punch, billet begins to fill the cavity. Due to the effect of friction between the billet and the punch, the middle of billet deforms firstly. In order to make the billet fully fill cavity, we need to apply a larger loading pressure at the later stage of precise forging forming. Thus, compared with precise forging, the overall uniformity of the tooth deformation is obviously improved and the filling effect and full degree are much better.

\subsection{The Flow Behavior}

above, the flow behavior of billet has a great difference between the two techniques because of the different loading methods. In order to make a further analysis, the metal flow behavior on the billet during rotary forging forming process is shown in Figure 4.

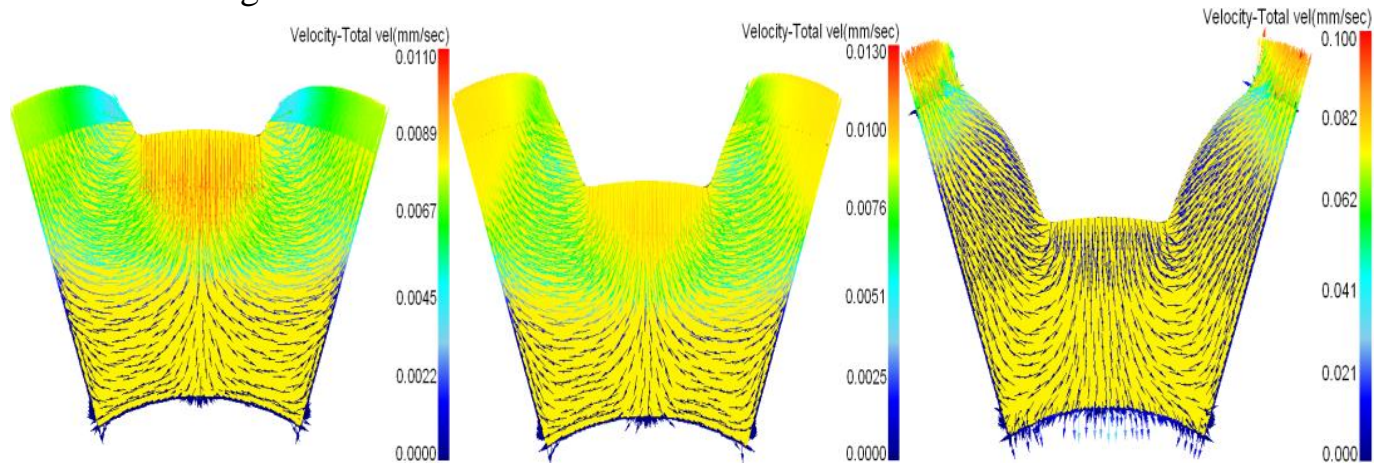

Figure 4. Comparison of Velocity Field Distribution

As is shown in Figure 4.the flow velocity of the billet lateral metal is relatively high, while the velocity is gradually reduces along the radial during the gear rotary forging process. Shunt surface is coincided with the central of the punch. When the punch moves along the radial direction, the bottom of the gear along the loading direction generates the deformation flow. Because of the extrusion of tooth root around the adjacent metal, the gear cavity is gradually filled with lots of metal until it is full. At the early stage of precise forging, the material flows along radial direction. At the same time, with the increase of punch's displacement, the "circular" flow interface moves along the axial direction gradually and most part of the billet flows outward. The material gradually converges toward the tooth cavity. The circular flow interface changes from "circular" to "radial" in the centre between two teeth and presents a tendency to flow around the shunt. Furthermore the billet height significantly reduces along the loading direction. A large amount of material flows in the cavity of the two sides of the shunt surface. In the final stage, the cavity is filled with metal and the excess metal begins to flow outside. As a result, velocity field on shunt surface recharges from "radial" to "circular". Compared with precise forging process, the complexity of the metal flow during the process of rotary forging is much smaller. According to the flow velocity field, the deformation of each part is obviously improved.

\subsection{Deformation Characteristics}

Due to the difference of deformation conditions, the stress states of billet subsequently change in the forming process. The effective stress of precise forging and rotary forging is shown in Figure 5. 


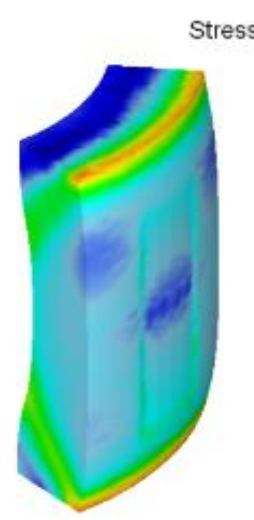

Initial Stage

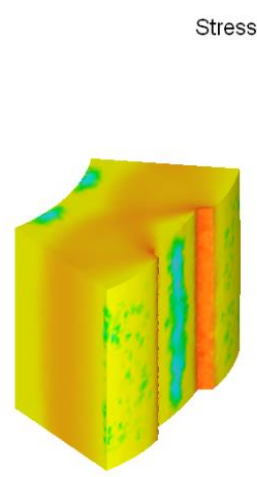

Initial Stage
tress-Effective(MPa)

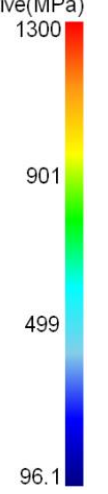

96.1

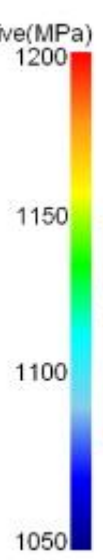

1150
1100
1050

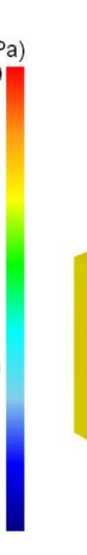

Medium Stage (a)Precise forging

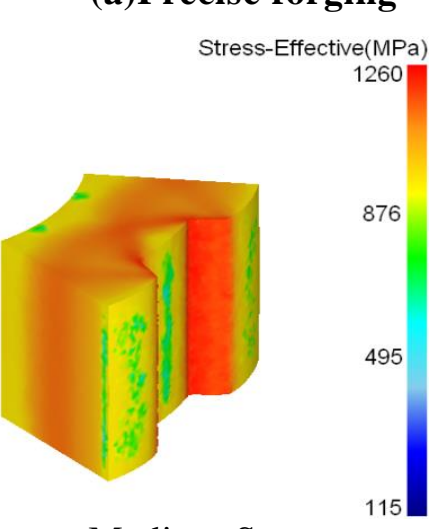

Medium Stage

(b)Rotary forging

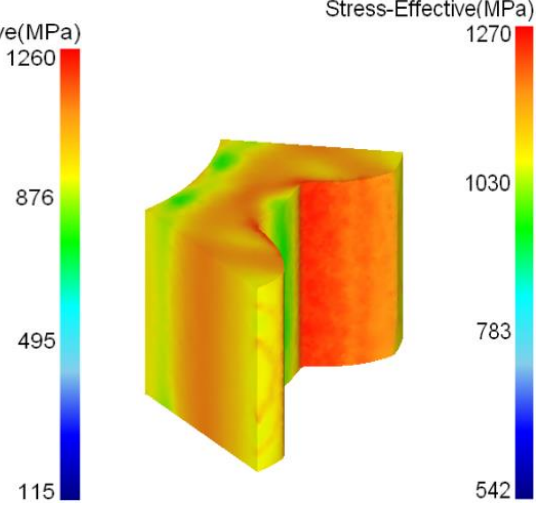

Final Stage

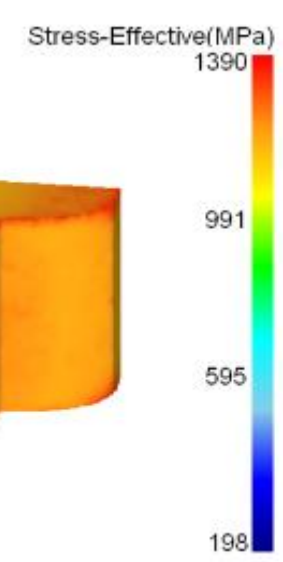

Final Stage

Figure 5. Comparison of Effective Stress Distribution

As is shown in Figure 5(a), in the early precision forging forming, the effective stress value on lateral angle of the ring billet is the biggest, where is the first place to yield and produce plastic deformation. There is an effect of friction between the punch and the billet, so plastic deformation of the inside corner will not occurred. With the movement of punch, the billet height decreases obviously and the cavity starts to be filled. At this point, the effective stress values of each part billet are significantly increased. The plastic deformation will not occur on the whole billet. The forming tooth apex angle is completed at the end of the filling, and then the effective stress value is the maximum.

The effective stress variation in the process of the rotary forging is shown in Figure (b). Because of the different loading methods, in the early rotary forging stage, the tooth cavity is going to be filled and the effective stress of the tooth root is the biggest. As the punch moves continuously along the radial, the plastic deformation at tooth root is significantly increased in rotary forging.

In order to compare the deformation characteristics of billet in the different forming methods, effective strain peak and the grid deformation in precision forging and rotary forging are compared in Figure 6. 


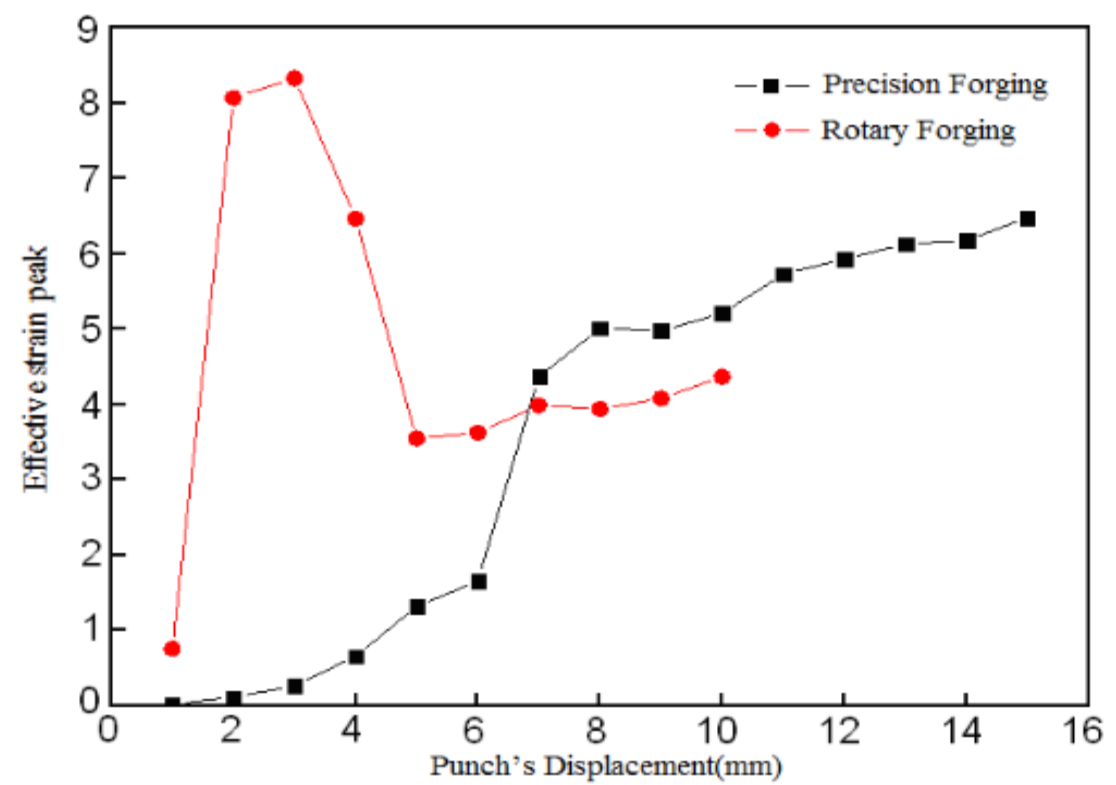

(a) Effective strain peak

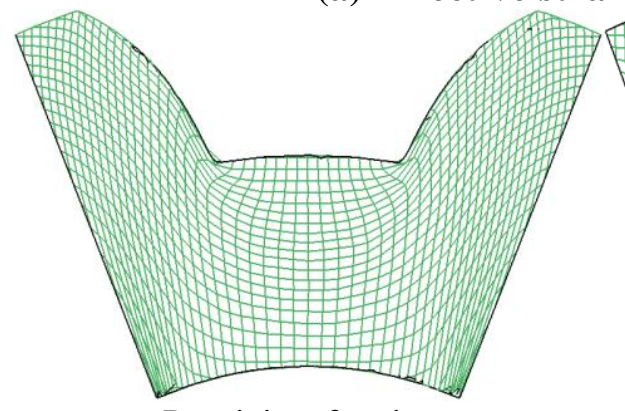

Precision forging

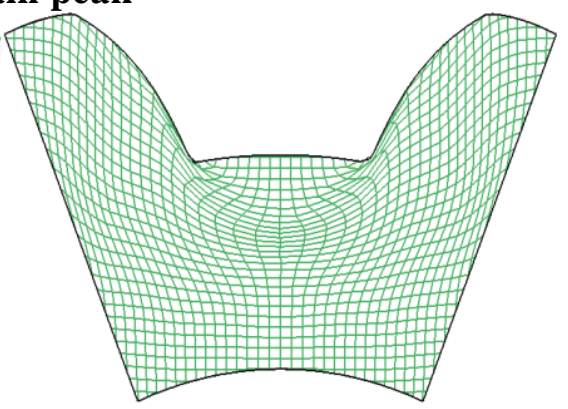

Rotary forging

(b) The grid changes

Figure 6. Deformation Characteristics of the Contrast

As shown in Figure 6(a),with the moving of punch, the effective strain peak on the billet show a continuous increase trend in the precision forging forming. The effective peak strain slightly increases in the middle forming. The trend of rotary forging effective peak strain is at first increases and then decreases, and increases at last.

As shown in Figure 6(b), the change characteristics of the grid are given along the cross section of gear. Because of the different forming methods, the clear differences between each part deformation behavior on the billet are much larger. Compared with the precision forging forming, the deformation uniformity of each part is significantly improved in the rotary forging.

\subsection{Displacement Load Changes}

Figure 7 shows the displacement-load curve in the process of rotary forging forming and precise forging. 


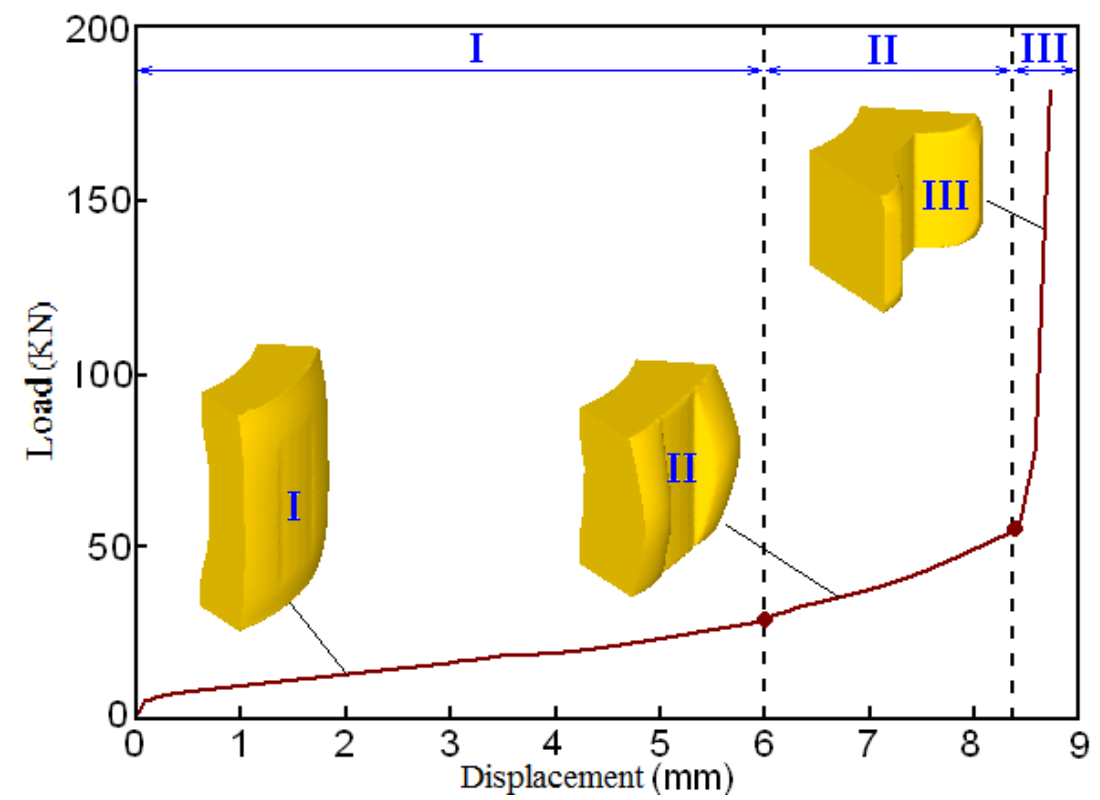

(a) Precision forging

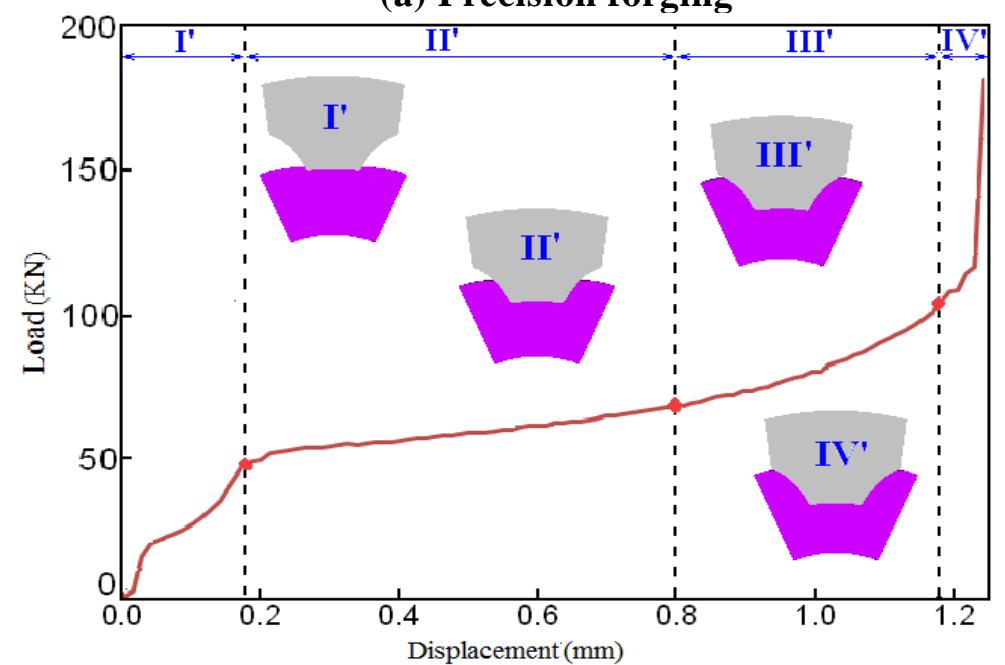

(b) Rotary forging

Figure 7. Displacement-Load Curve under Different Technological Conditions

As is shown in Figure 7(a), the gear precision forging forming process can be roughly divided into three stages: upsetting (I), direct extrusion (II),corner filling (III).In the early forming stage, the punch begins to contact the billet and appears compressive deformation and it makes the contact area increase between the upper and bottom of the billet. Along the height direction, the billet size in this stage is significantly decreased, whiles the circular and radial is significantly increased. Because of the existence of the contact part, the velocity of the metal near the upper and lower contact surface is obviously lagging behind the middle. After compress deformation, the deformation of the billet is characterized by a single drum. The free surface of compressive deformation billet is always a little larger, so the forming load increases almost linearly. In the middle forming stage, the contact area between the billet and cavity wall is increasing significantly. At this time, the billet is gradually filling into the cavity and the forming load continuously increases. At the end of forming stage, most of the cavity is completely filled and the metal deformation decreases significantly. At this time, the billet has little free surface. In 
order to fill the cavity tooth angle, we need to increase the forming load to make the amplification quickly increase.

The gear rotary forging load can be roughly divided into four stages: open punching (I'), backward extrusion (II'), forward extrusion (III'), corner filling (IV'), as is shown in Figure 7.In the initial forming period, the billet free surface is very large. The load is applied on the billet along the radial direction and the deformation characteristics are similar to the open punching. The material that contacts punch appears compression and flowing deformation with the forming load increasing. The middle forming period can be divided into two stages. The billet experienced compression deformation and gradually filled towards the cavity area. Compared with the contact part between the punch and the billet, the free surface in the root of the teeth is much bigger and the resistance is increasing gradually with the filling of the cavity. Therefore, the change trend of the forming load in this stage is firstly reduces and then increases. In the end forming stage, the teeth angle can be completely filled under a larger loading condition, so the load continuously increases and the increase become much bigger.

\section{Conclusion}

1. The technique of rotary forging technology is much better than the traditional gear precision forging relied on the fixed form mode along the axial loading. Rotary forging technology uses the punch with the same gear module and can carry out simultaneously radial loading. That is, the loading method changes from integral loading to local loading. Therefore, the forming load can be significantly reduced.

2. In the gear rotary forging forming technique, the punch moves along the radial direction and a convex gear can be directly formed. The billet along the axial direction almost simultaneously fills the cavity at various points. Some problems can be avoided during precision forging forming. The strength of the gear can be significantly improved while the loads can be reduced.

3. Compared with precision forging, rotary forging can significantly shorten the actual stroke of the punch. The complexity of the billet flowing deformation is significantly decreased. The deformation uniformity and the forming quality are improved.

\section{Acknowledgments}

This work was financially supported by the National Natural Science Foundation of China $(51375124,51575148)$, the Natural Science Foundation of Heilongjiang Province of China(E2016048).

\section{References}

[1] C. Choia and Y. Choib, International Journal of Machine tools \&Manufacture, vol. 39, (1999), pp. 15-75.

[2] A. Behrensa, E. Doegea,S. Reinschb, K. Telkampb, H. Daehndela and A. Speckerb, Journal of Materials Processing Technology, vol. 185, (2007), pp. 139.

[3] Hyatta, M. Piberb, N. Chaphalkara, O. Kleinhenza and M. Moria, Procedia CIRP, vol. 14, (2014), pp. 72.

[4] J. Choi, H. Y. Cho, C. Y. Jo, Journal of Materials Processing Technology, (2000), vol. 104, pp. 67.

[5] H. Sadeghia and T.A. Deanb,Journal of Materials Processing Technology, vol. 45, (1994), pp. 25.

[6] L. Hua, K.S Wang and Q.K Liu, Journal of Materials Processing Technology, vol. 187, (2007), pp. 60.

[7] M.L. Alvesa, J.M.C. Rodriguesb, P.A.F. Martins, Finite Elements in Analysis and Design, vol. 6, (2001), pp. 5-49. 